ECIR tutorial part 1 and 2 Monday, April 6, 2009

\title{
Information Extracting and Linking in a Retrieval Context
}

Marie-Francine Moens

Katholieke Universiteit Leuven

Belgium
Djoerd Hiemstra

University of Twente

The Netherlands

http://www.cs.kuleuven.be/ sien/ http://www.cs.utwente.nl/ hiemstra/ 


\section{Overview of the tutorial}

1. Introduction and motivation

2. Information extraction and linking techniques

Coffee break

3. Probabilistic models for information retrieval

4. Models for entity ranking 


\section{PART 1 \\ Introduction and Motivation}




\section{Motivation}

- In the fields of natural language processing, computer vision and audio processing, we develop techniques for content recognition, often called information extraction

- In the field of data mining, linking equivalent or related content is a hot topic

- The results are often of probabilistic nature

- How can we use these results of these developments in a (probabilistic) information retrieval model? 


\section{Information extraction}

"Information extraction is the identification, and consequent or concurrent classification and structuring into semantic classes, of specific information found in unstructured data sources, such as natural language text, providing additional aids to access and interpret the unstructured data by information systems."

[Moens 2006] 


\section{Information extraction from text}

- Tested in ARPA's Tipster Text Program and in the past Message Understanding Conferences (MUC), Automatic Content Extraction (ACE), current Text Analysis Conference (TAC) (National Institute of Standards and Technology, NIST) and Computational Natural Language Learning (CoNLL) 


\section{Information extraction from text}

- Named entity recognition (e.g., person names, locations, organizations, ...)

- Opinion extraction (e.g., positive, negative feelings expressed, ...)

- Event extraction:

- Topics of an event and the entities involved

- Extraction of details of an event:

- e.g., type of event, time, location, number of victims, ... 


\section{Information extraction from text}

- Extraction of information on Web page:

- e.g., e-mail, date of availability of a product, ...

- Extraction of scientific data from publications:

- e.g., localization of a gene, treatment of a disease, function of a gene, ...

- AND NOT ONLY TEXT !!! 


\section{Birthday party}

\section{Happyness}

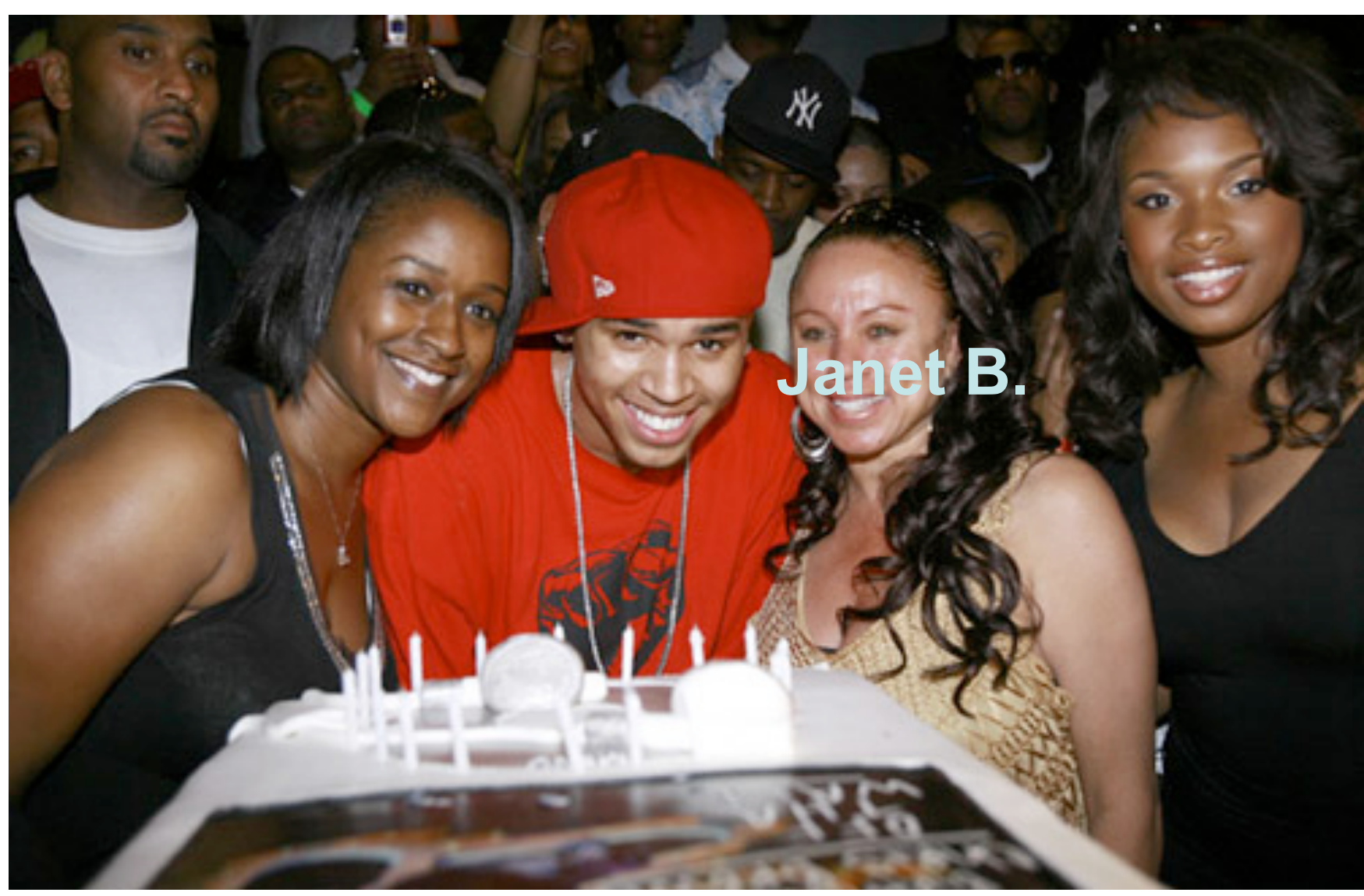

Cake

Candles 


\section{Latent semantic topic models}

- = a class of unsupervised (or semi-supervised) models in which the semantic properties of words and documents are expressed in terms of topics

- models are also called aspect models

- Latent Semantic Indexing:

- the semantic information can be derived from a worddocument matrix

[Deerweester et al. 1990]

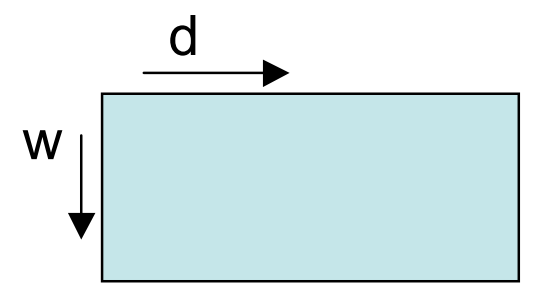

II - But, LSI is unable to capture multiple senses of a word

- Probabilistic topic models 


\section{Probabilistic topic model}

- = Generative model for documents: probabilistic model by which documents can be generated

- document $=$ probability distribution over topics

- topic $=$ probability distribution over words

- To make a new document, one chooses a distribution over topics, for each topic one draws words according to a certain distribution:

- select a document $d_{j}$ with probability $P\left(d_{j}\right)$

- pick a latent class $z_{k}$ with probability $P\left(z_{k} \mid d_{j}\right)$

- generate a word $w_{i}$ with probability $P\left(w_{i} \mid z_{k}\right)$ [Steyers \& Griffiths 2007] 


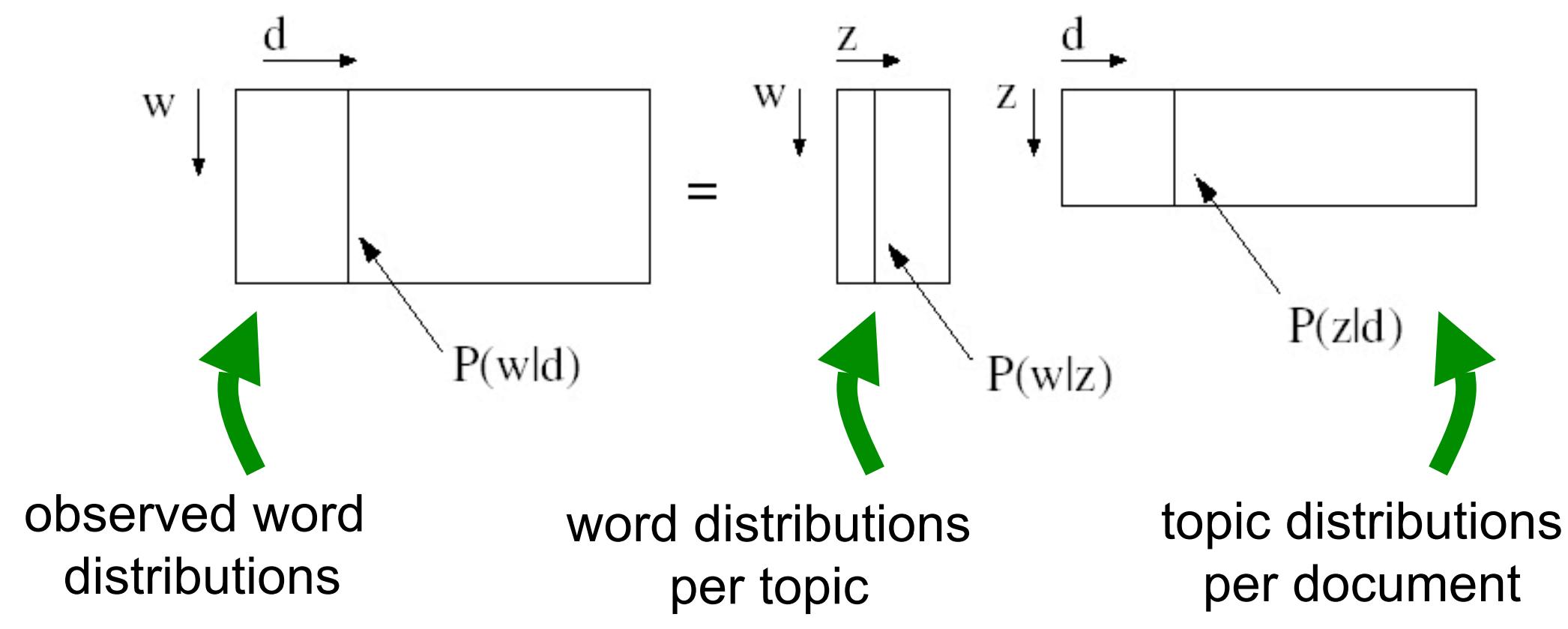

=> Popular model for document representation 


\section{Linking}

- Links between content that is connected

- Might be equivalence links

- Or just reference links to related information

- And many other types ...

- Often focused on persons: e.g., Web People Search competition 


\section{In one document}

\section{- Noun phrase coreference resolution:}

Pakzad is one of several Afghan women's rights advocates who are in the U.S. Capit al this week to address a move the Obama administration is considering: reaching out to Taliban moderates in an effort to bring peace to Afghanistan.

She took part in a hearing on Capitol Hill -- sponsored by Sens. Mary Landrieu, D-Louisiana, and Richard Lugar, R-Indiana -- titled "Women Shaping Afghanistan'...

[CNN, World News, 11-3-2009] 


\section{Across documents}

\section{Tom Mitchell}

Fredkin Professor of AI and Machine Learning

Chair, Machine Learning Department

School of Computer Science

Carnegie Mellon University

412-268-2611, Tom.Mitchell@cmu.edu, Resume, A personal interview

Assistant: $\underline{\text { Sharon Cavlovich, }} 412$ 268-5196

What is Machine Learning, and where is it headed?

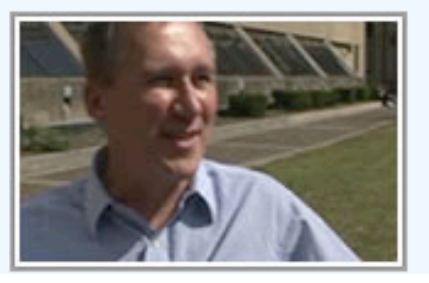

\section{Tom Mitchell}

\section{Tom Mitchell}

Research Fellow

Vulnerability and Poverty Reduction Team

Climate Change and Disasters Group

CV (Word)

Tel: $44(0) 1273915757$

E-mail: t.mitchell@ids.ac.uk

Administrative contact: Hannah Bywaters

(h.bywaters@ids.ac.uk)

Biography

Dr. Tom Mitchell is a Research Fellow at IDS, having previously been a

member of the Benfield UCL Hazard Research Centre. He specialises in

climate change adaptation and disaster risk reduction. His interests include

pro-poor climate and disaster governance and he co-ordinates the children

in a changing climate programme. 


\section{Across documents}

...Woodward's source in the

Plame scandal

\section{... senior administration official ...}

... Richard Armitage ... 


\section{Across media}

\section{YAHOO! NEWS}

News Photos - News Home - Help

World reacts to U.S. inauguration

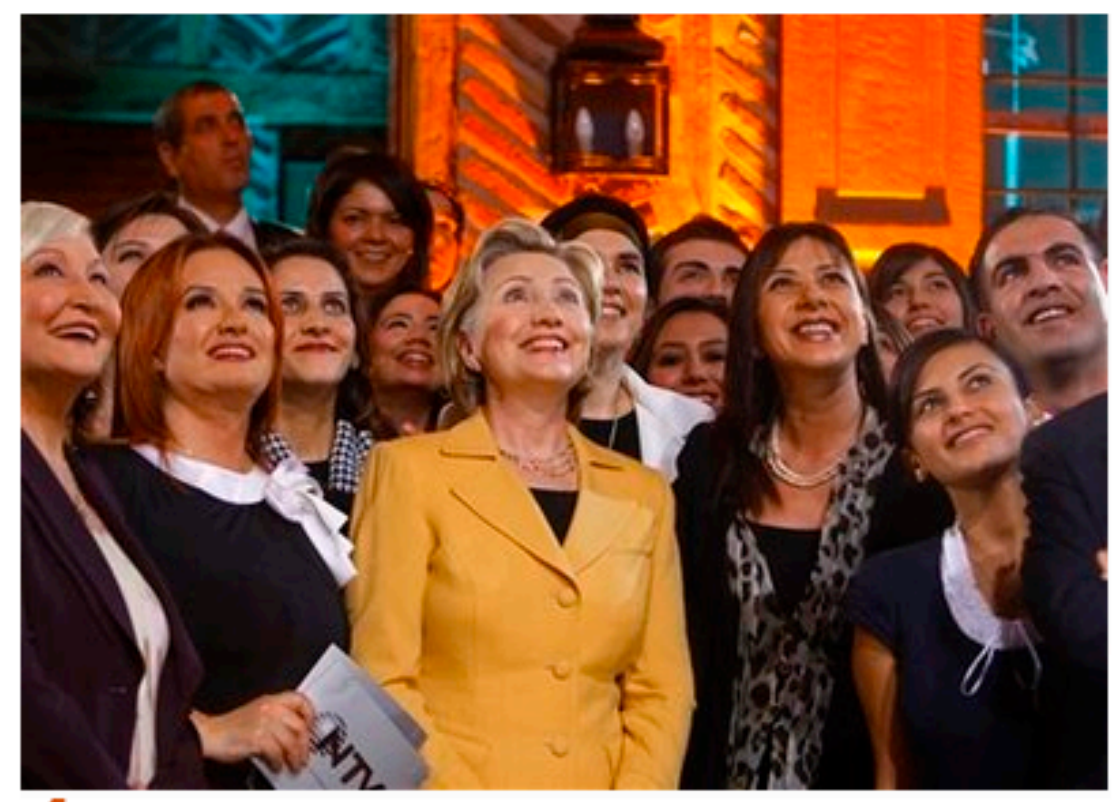

4.P Associated Press
Thu Mar 12, 10:46 PM ET

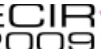




\section{Applications in IR}

- Information extraction and linking: has received during decades a large interest because of its potential for information access, mining, summarization, ...

- In IR context: interest is boosted

- Already popular:

- People/expert search

- Opinion search

- Event search

- Other types of search follow: Concept search 


\section{People and expert search}

- Finding people ca. $5 \%$ of all Web queries

- Finding experts on a certain topic useful in business, educational and other settings 


\section{Opinion search}

- E.g., monitoring and analyzing social media such as blogs and discussion forums => online business intelligence, intelligence informatics, ... 


\section{Event search}

- Very traditional IE

- Tasks:

- Named entity recognition

- Noun phrase coreference resolution

- Entity relation recognition

- Event recognition (who, what, where, when) 


\section{Nastia Liukin wins women's gymnastics all-around gold}

Adjust font size:

Nastia Liukin of the United States edged her compatriot Shawn Johnson to win the women's allaround after a breathtaking Olympic gymnastics competition on Friday.

\section{Wirity one-two on the podium, the American duo let} the hosts' gold rush in the gymnastics pause after China wrapped up all the first three titles (men's team, women's team, men's all-round) in Nationgrium.

Liukin collected 63.325 points, after flawless exercises on each of the four apparatus with a good combination of

difficulty and quality, beating Johnson by 0.600 . The Narge medal went to Yang Yilin of China in 62.650.

Coming out in her first Olympic Games, the 18-year-old Liukin struck the mostrareted-acls madal which she had waited for years.

She was an unlucky runner-up in the 2005 world championship in Melbourne, beaten by Chellsie Memmel by 0.001 points in her debut to the international arena, and the title also eluded her in the following two chamoionshios.

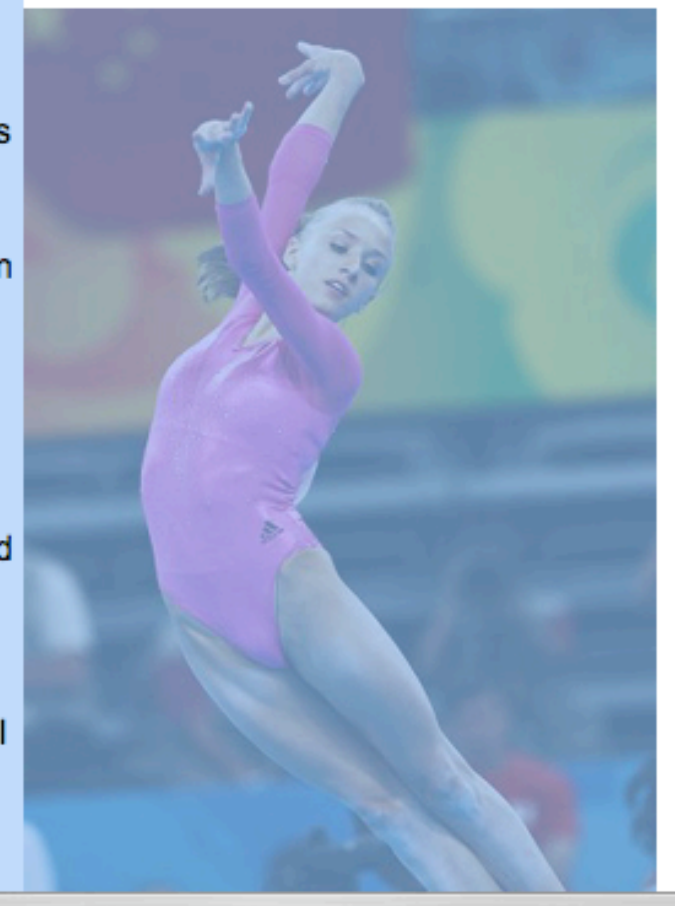




\section{Concept search}

- Especially popular in multimedia search:

- Low level features (e.g. of image frames in video) are described with conceptual terms

- Concepts are often defined with terms from an ontology

- Or, repeating patterns in the data can be considered as "concept"

- Less developed in text search, except for specific domains such as the legal field 
- How do we successfully extract and link information?

- How do we integrate the results in a (probabilistic) retrieval model? 


\section{PART 2 \\ Information Extraction and Linking Techniques}




\section{Overview}

- Information extraction from text:

- Named entity recognition

- Opinion extraction

- Event extraction

- Topic modeling (text, images, ...)

- Linking of content

- One document

- Across documents

- Across media 


\section{Information extraction}

- Most successful techniques:

- Machine learning techniques that train from training set annotated

- Training and test examples are usually represented as a vector of features

- From text: linguistic properties, further called feature functions $f$, that can be exploited:

- Lexical features

- Morpho-syntactic features

- Semantic features

- Discourse features 


\section{Named entity recognition}

- Named entity recognition recognizes and classifies named expressions in text (such as person, company, location or protein names):

Example:

John Smith works for IBM.

Person Company 


\section{Named entity recognition}

- Two problems: Segmentation + Classification

- Constituent based processing:

- Constituents are first identified (constituency parser or phrase chunker)

- Constituents are classified

- Use of BIO format:

- $\mathrm{B}=$ Begin, $\mathrm{I}=$ Inside, $\mathrm{O}=$ Outside labels per class

- Words or tokens are classified

Here illustrated for NER, but similar approach for other

extraction tasks (e.g., relation extraction) 
Table 4.1. Typical features in a named entity recognition task of the candidate entity name $i$ that occur in the context window of $l$ words.

\begin{tabular}{|l|l|l|}
\hline FEATURE & VALUE TYPE & VALUE \\
\hline Short type & Boolean & $\begin{array}{l}\text { True if } i \text { matches the short type } j \text {; False } \\
\text { otherwise. }\end{array}$ \\
\hline POS & Nominal & $\begin{array}{l}\text { Part-of-speech tag of the syntactic head of } \\
i .\end{array}$ \\
\hline Context word & $\begin{array}{l}\text { Boolean or real } \\
\text { value between } 0 \\
\text { and 1; } \\
\text { Or nominal. }\end{array}$ & $\begin{array}{l}\text { True if the context word } j \text { occurs in the } \\
\text { context of } i \text {; False otherwise; If a real } \\
\text { value is used, it indicates the weight of the } \\
\text { context word } j . \text { Alternatively, the context } \\
\text { word feature can be represented as one } \\
\text { feature with nominal values. }\end{array}$ \\
\hline POS left & Nominal & $\begin{array}{l}\text { POS tag of a word that occurs to the left of } \\
i .\end{array}$ \\
\hline POS right & Nominal & $\begin{array}{l}\text { POS tag of a word that occurs to the right } \\
\text { of } i .\end{array}$ \\
\hline $\begin{array}{l}\text { Morphological } \\
\text { prefixes/suffixes }\end{array}$ & Nominal & Prefix or suffix of $i$. \\
\hline & & \\
\hline
\end{tabular}




\section{Classification}

Maria Sharapova wrote an article in the New York Times

$\begin{array}{cccc}\boldsymbol{x}_{1} & \boldsymbol{x}_{2} & \boldsymbol{x}_{3} & \boldsymbol{x}_{4} \\ y_{1} & y_{2} & y_{3} & y_{4}\end{array}$

- Adhering to maximum entropy principle because of incomplete training data, e.g., maximum entropy classifier, conditional random field

- Context dependent classification:

- Content cannot exist without other content, or has a large chance to occur with it, e.g., conditional random field 


\section{Logistic regression}

- We predict a ratio of two probabilities as the log odds (or logit) function:

$$
\operatorname{logit}(p(x))=\ln \left(\frac{p(x)}{(1-p(x))}\right)
$$

- Logistic regression: model of regression in which we use a linear function to estimate the logit of the probability

- Linear function given $k$ feature functions $f$ and $a$ weight vector $w$ :

$$
\sum_{k} w_{i} \times f_{i}=w \cdot f
$$




\section{Logistic function}

$$
\begin{array}{ll}
\ln \left(\frac{p(y=\text { true } \mid x)}{1-p(y=\text { true } \mid x)}\right)=w \cdot f & \begin{array}{l}
\text { Maps values } \\
\text { from }-\infty \text { to }+\infty \\
\text { to a probability }
\end{array} \\
\frac{p(y=\text { true } \mid x)}{1-p(y=\text { true } \mid x)}=e^{w \cdot f}=\exp (w \cdot f) & p(y=\text { false } \mid x)=\frac{1}{1+e^{w \cdot f}} \\
p(y=\text { true } \mid x)+p(y=\text { true } \mid x) e^{w \cdot f}=e^{w \cdot f} & p(y=\text { false } \mid x)=\frac{e^{-w \cdot f}}{1+e^{-w \cdot f}} \\
p(y=\text { true } \mid x)=\frac{e^{w \cdot f}}{1+e^{w \cdot f}} \quad p & 1
\end{array}
$$




\section{Logistic regression classification}

New example can be labeled true if:

$$
\begin{aligned}
& p(y=\text { true } \mid x)>p(y=\text { false } \mid x) \\
& \frac{p(y=\text { true } \mid x)}{p(y=\text { false } \mid x)}>1 \\
& \frac{p(y=\text { true } \mid x)}{1-p(y=\text { true } \mid x)}>1 \\
& e^{w \cdot f}>1 \\
& w \cdot f=\sum_{k} w_{k} f_{k}>0
\end{aligned}
$$




\section{Logistic regression training}

- Learning a logistic regression function can be seen as learning a hyperplane which separates points in space that are in the positive class from points that are not in the class

- Training is learning the weights so that the probability of the observed values $y$ is the highest: through convex optimization:

- Several methods: quasi-Newton methods, gradient ascent, conjugate gradient and various iterative scaling algorithms 


\section{Maximum entropy modeling}

- Maxent deals with a larger number of classes: multinomial logistic regression

- Let there be $C$ different classes: $y_{1}, y_{2}, \ldots, y_{C}$

- We estimate the probability that $y$ is a particular class $c$ given $k$ feature functions $f$ as:

$$
\begin{gathered}
p(y \mid x)=\frac{1}{Z} \exp \sum_{k} w_{i} f_{i} \\
p(y \mid x)=\frac{\exp \sum_{k} w_{i} f_{i}(y, x)}{\sum_{y^{\prime} \in C} \exp \sum_{k} w_{i} f_{i}\left(y^{\prime}, x\right)}
\end{gathered}
$$


- [Berger et al. CL 1996] showed that the exponential model for multinomial logistic regression when trained according to the maximum likelihood criterion, also finds the maximum entropy distribution subject to the constraints from the feature functions 


\section{Conditional random fields}

- Let $\boldsymbol{X}$ be a random variable over data to be labeled and $\boldsymbol{Y}$ a random variable over corresponding labels

- All components $\boldsymbol{Y}_{\boldsymbol{j}}$ of $\boldsymbol{Y}$ are assumed to range over a finite label alphabet $\sum$

- A conditional random field is viewed as an undirected graphical model or Markov random field, conditioned on $\boldsymbol{X}$

- We define $G=(V, E)$ to be an undirected graph such that there is a node $v \in V$ corresponding to each of the random variables representing an element $Y_{v}$ of $\boldsymbol{Y}$

- If each random variable $Y_{v}$ obeys the Markov property with respect to $G$, then the model $(\boldsymbol{Y}, \boldsymbol{X})$ is a conditional random field 


\section{Conditional random fields}

- In theory the structure of graph $G$ may be arbitrary, however, when modeling sequences, the simplest and most common graph structure encountered is that in which the nodes corresponding to elements of $\boldsymbol{Y}$ form a simple firstorder Markov chain (linear-chain CRF)

- Note: in the following $\boldsymbol{x}$ refers to an observation sequence and not to a feature vector and $y$ to a labeling sequence 


\section{Conditional random fields}

- Named entity recognition:

- Sequence of observations: $\boldsymbol{x}=\left(\boldsymbol{x}_{1}, \ldots, \boldsymbol{x}_{T}\right)$

- Sequence of semantic labels: $y=\left(y_{1}, \ldots, y_{T}\right)$

- Sequence forms first order Markov chain

- A novel observation sequence $\boldsymbol{x}$ is labeled with $y$, so that the conditional probability $p(y \mid x)$ is maximized:

$$
p(y \mid \boldsymbol{x})=\frac{1}{Z} \exp \left(\sum_{i=1}^{k} \sum_{j=1}^{T} w_{i} f_{i}\left(y_{j}-1, y_{j}, \boldsymbol{x}, j\right)\right)
$$

- Trained with dynamic programming techniques that are similar to the Baum-Welch algorithm [Lafferty et al. ICML 2001] 


\begin{tabular}{|c|c|c|c|c|c|c|}
\hline \multicolumn{7}{|c|}{ F1 scores on the CoNLL Dataset } \\
\hline Approach & LOC & ORG & MISC & PER & ALL & Relative Error reduction \\
\hline \multicolumn{7}{|c|}{ Bunescu and Mooney (2004) (Relational Markov Networks) } \\
\hline Only Local Templates & - & - & - & - & 80.09 & \\
\hline Global and Local Templates & - & - & - & - & 82.30 & $11.1 \%$ \\
\hline \multicolumn{7}{|c|}{ Finkel et al. (2005)(Gibbs Sampling) } \\
\hline Local+Viterbi & 88.16 & 80.83 & 78.51 & 90.36 & 85.51 & \\
\hline Non Local+Gibbs & 88.51 & 81.72 & 80.43 & 92.29 & 86.86 & $9.3 \%$ \\
\hline \multicolumn{7}{|c|}{ Our Approach with the 2 -stage CRF } \\
\hline Baseline CRF & 88.09 & 80.88 & 78.26 & 89.76 & 85.29 & \\
\hline + Document token-majority features & 89.17 & 80.15 & 78.73 & 91.60 & 86.50 & \\
\hline + Document entity-majority features & 89.50 & 81.98 & 79.38 & 91.74 & 86.75 & \\
\hline + Document superentity-majority features & 89.52 & 82.27 & 79.76 & 92.71 & 87.15 & $12.6 \%$ \\
\hline + Corpus token-majority features & 89.48 & 82.36 & 79.59 & 92.65 & 87.13 & \\
\hline + Corpus entity-majority features & 89.72 & 82.40 & 79.71 & 92.65 & 87.23 & \\
\hline (All features) & 89.80 & 82.39 & 79.76 & 92.57 & 87.24 & $13.3 \%$ \\
\hline
\end{tabular}

Named entity recognition: 2-stage approach: 1) CRF with local features; 2) local information and output of first CRF as features. Comparison against competitive approaches. Baseline results are shown on the first line of each approach.

[Krishnan \& Manning 2006]

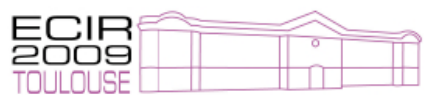




\section{Opinion extraction}

- Web documents: forum for:

- Exchange of opinions

- Dissemination of propaganda

- => extracting opinions or sentiments is of interest for businesses, governments, ... 


\section{Opinion extraction}

- Learning emotion patterns in blog texts:

- Positive, negative and neutral feeling

- Problems:

- Large variety of expressions (noisy texts !!!) and relatively few annotated examples

- Emotion is attributed to an entity

- How to reduce annotation?

- Language/domain portability 
The movie really seems to be spilling the beans on a lot of stuff we didnt think we hand - if this is their warm up, what is going to get us frothing in December

de grote merken mogen er dan patserig uitzien en massa's pk hebben maarals de bomen wat dicht bij elkaar staan en de paadjes steil enbochtig,dan verkies ik mijn Jimny.

L'é tro bel cet voitur Voici tt ce ki me pasione ds ma petite vi!!!é tt mé pote é pll dotre truk!!!Avou de Dcouvrir 


\section{Opinion extraction}

- Solutions tested:

- Feature selection or extraction

- Single classifier versus a cascaded classifier versus bagged classifiers

- Active learning

[Abassi et al. TOIS 2008]

[Boiy \& Moens IR 2009] 
Table IV. English and Arabic Feature Sets

\begin{tabular}{|c|c|c|c|c|}
\hline Category & Feature Group & English & Arabic & Examples \\
\hline \multirow{4}{*}{ Syntactic } & POS N-grams & varies & - & frequency of part-of-speech tags (e.g., NP VB) \\
\hline & Word Roots & - & varies & frequency of roots (e.g., slm, ktb) \\
\hline & Word $\mathrm{N}$-grams & varies & varies & word n-grams (e.g. senior editor, editor in chief) \\
\hline & Punctuation & 8 & 12 & occurrence of punctuation marks (e.g., !;:, ?) \\
\hline \multirow[t]{10}{*}{ Stylistic } & Letter N-Grams & 26 & 36 & frequency of letters (e.g., a, b, c) \\
\hline & Char. N-grams & varies & varies & character n-grams (e.g., abo, out, ut, ab) \\
\hline & Word Lexical & 8 & 8 & total words, $\%$ char. per word \\
\hline & Char. Lexical & 8 & 8 & total char., $\%$ char. per message \\
\hline & Word Length & 20 & 20 & frequency distribution of $1-20$ letter words \\
\hline & Vocab. Richness & 8 & 8 & richness (e.g., hapax legomena, Yule's K) \\
\hline & Special Char. & 20 & 21 & occurrence of special char. (e.g., @ $\left.\# \$ \% \%^{\wedge} \&^{*}\right)$ \\
\hline & Digit N-Grams & varies & varies & frequency of digits (e.g., 100, 17, 5) \\
\hline & Structural & 14 & 14 & has greeting, has url, requoted content, etc. \\
\hline & Function Words & 250 & 200 & frequency of function words (e.g., of, for, to) \\
\hline
\end{tabular}

[Abassi et al. TOIS 2008] 


\section{Opinion extraction}

- Many studies use machine learning techniques that train from classified examples:

- Support vector machines and naive Bayes classifiers are very popular

- Link analysis techniques

- Active learning to select examples to annotate

- Augmented with linguistic rules of a language 


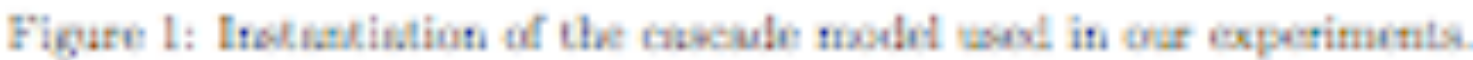

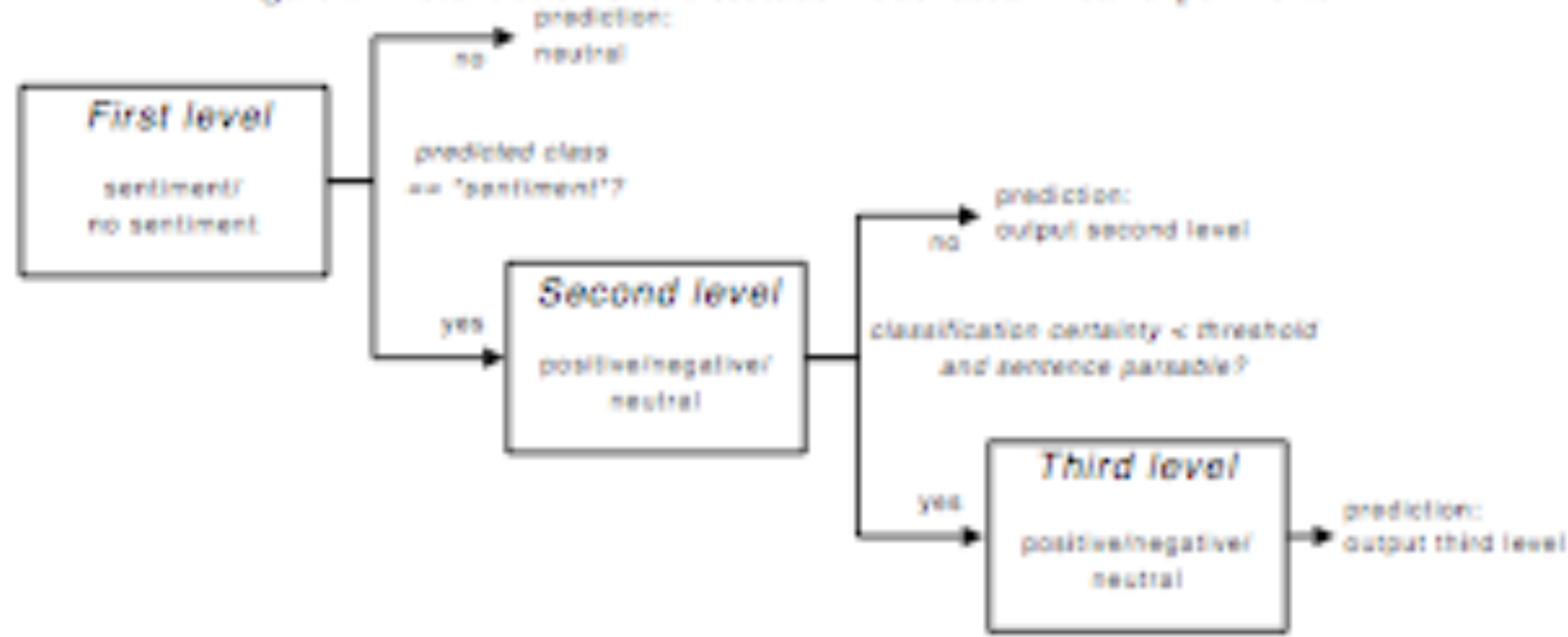

[Boiy \& Moens IR 2009] 


\section{Event extraction}

- Event = happening at a certain place at a certain time

- Involves named entities

- Involves topics

- Can be expressed in sentence

- Can be expressed in discourse

=> Named entities combined with topic models 


\section{Probabilistic Latent Semantic Analysis (pLSA)}

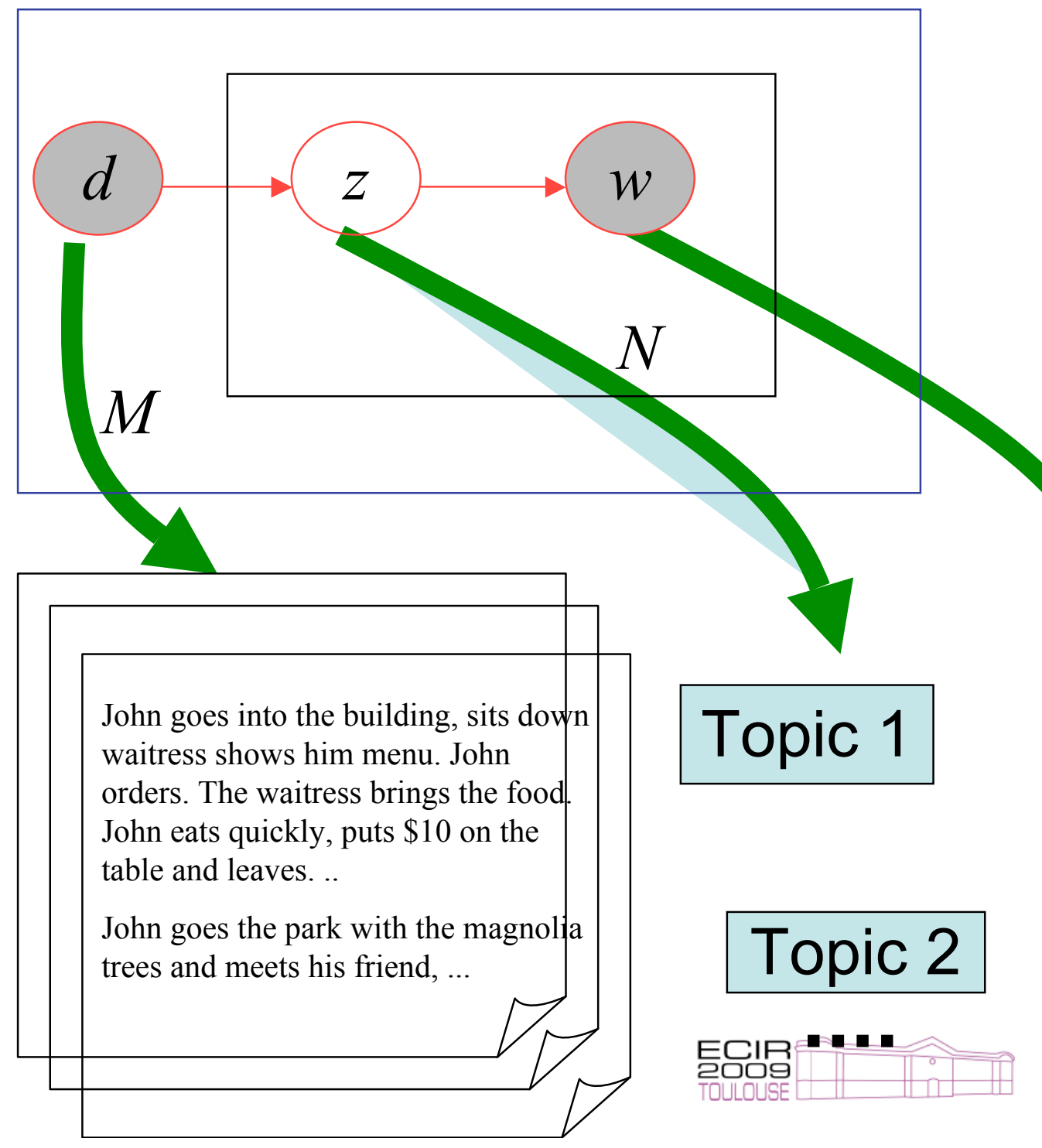

[Hofmann 1999]

$M=$ number of documents $N=$ number of words

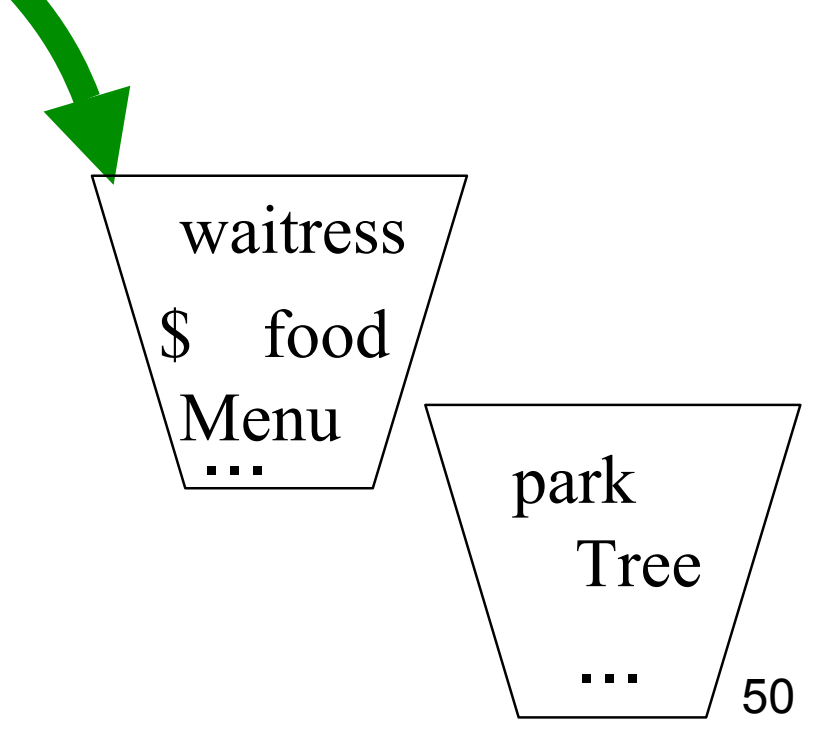




\section{Probabilistic Latent Semantic Analysis}

- Translating the document or text generation process into a joint probability model:

$$
\begin{aligned}
& P\left(d_{j}, w_{i}\right)=P\left(d_{j}\right) P\left(w_{i} \mid d_{j}\right) \\
& \text { where } \quad P\left(w_{i} \mid d_{j}\right)=\sum_{k=1}^{K} P\left(w_{i} \mid z_{k}\right) P\left(z_{k} \mid d_{j}\right) \\
& K=\text { number of topics (a priori defined) }
\end{aligned}
$$

Training:

maximizing

$$
L=\prod_{j=1}^{M} \prod_{i=1}^{N} P\left(d_{j}, w_{i}\right)^{n\left(d_{j}, w_{i}\right)}=\sum_{j=1}^{M} \sum_{i=1}^{N} n\left(d_{j}, w_{i}\right) \log P\left(d_{j}, w_{i}\right)
$$

where $n\left(d_{j}, w_{i}\right)=$ frequency of $w_{i}$ in $d_{j}, N=$ number of words in the vocabulary (e.g. trained with EM algorithm) 

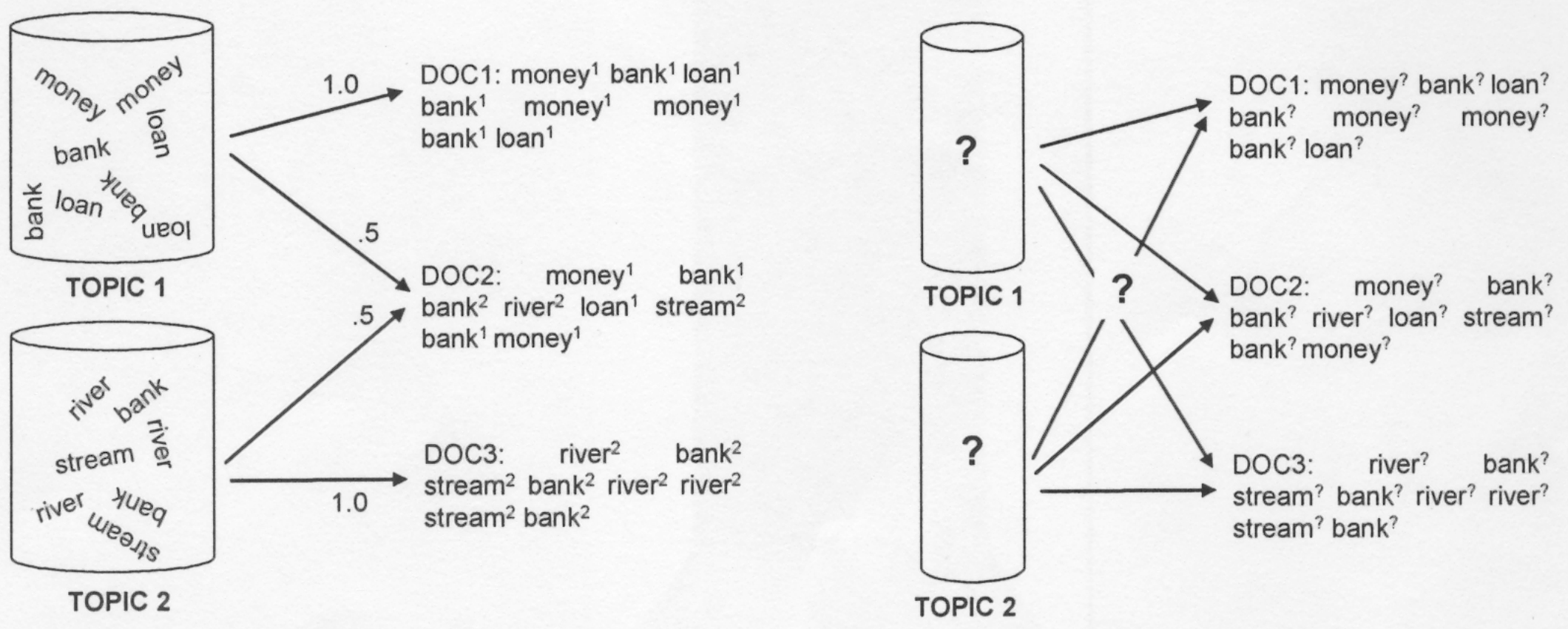

Figure 2. Illustration of the generative process and the problem of statistical inference underlying topic models

[Steyvers \& Griffiths 2007] 


\section{Latent Dirichlet Allocation}
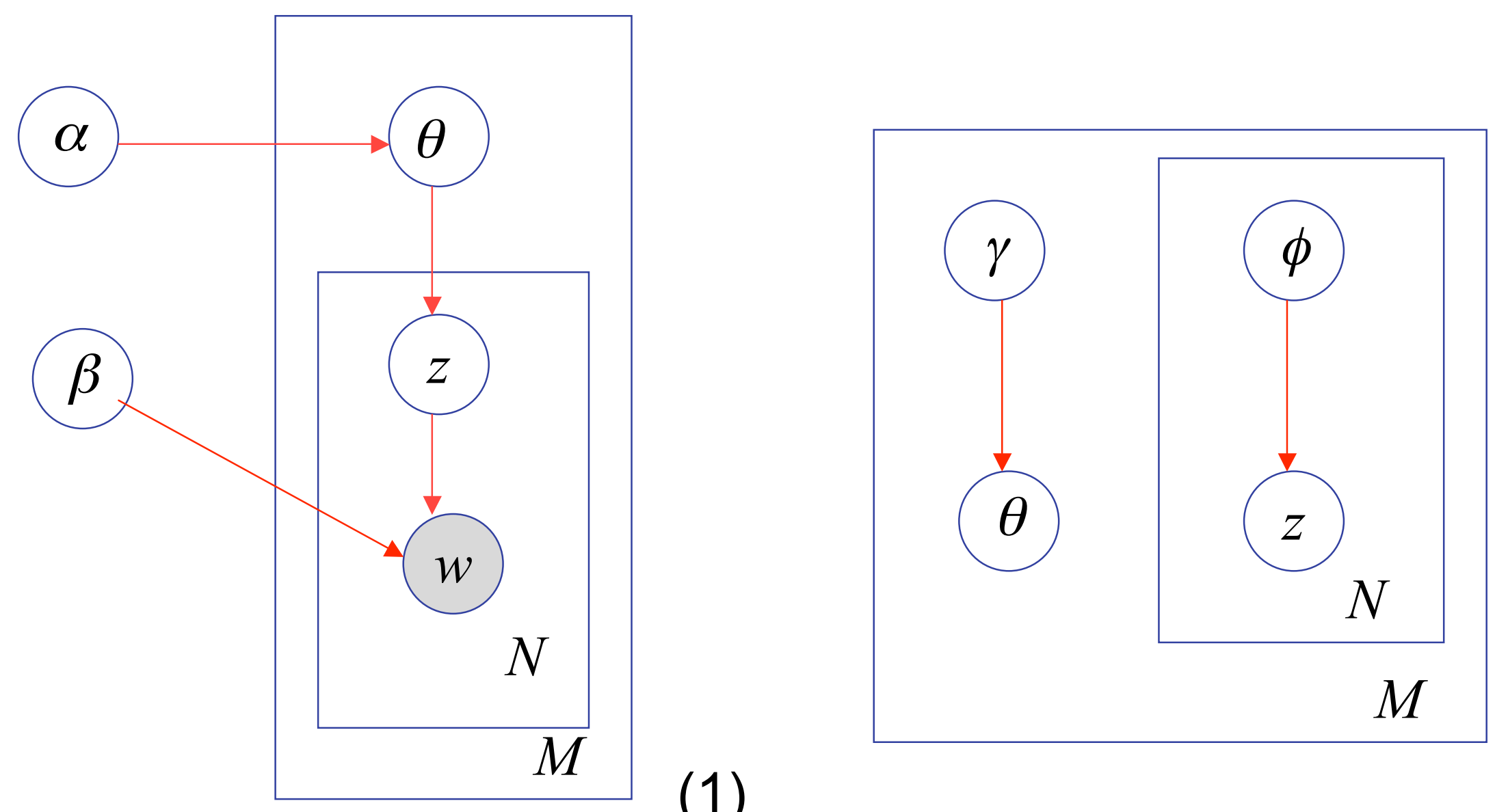

(1)

[Blei et al. JMLR 2003] 


\section{Latent Dirichlet Allocation}

- pLSA:

- learns $P\left(z_{k} \mid d_{j}\right)$ only for those documents on which it is trained except for some limited folding in

- number of hidden variables to learn grows linearly with the growth of the number of documents

- Latent Dirichlet Allocation (LDA) treats topic mixture weights as a Kparameter hidden random variable $\theta$

- Training

- Key inferential problem: computing the distribution of the hidden variables $\theta$ and $z$ given a document, i.e., $p(\theta, \mathbf{z} \mid \mathbf{w}, \alpha, \beta)$ : intractable for exact inference (model 1)

- $\alpha$ : Dirichlet prior, can be interpreted as a prior observation count for the number of times a topic is sampled in a document, before having observed any actual words from that document 


\section{Latent Dirichlet Allocation}

- Model $2=$ simple modification of the original graphical model 1: the chain $\alpha \rightarrow \theta \rightarrow z$ is replaced by $\gamma \rightarrow \theta$ and $\phi \rightarrow z$

- Compute approximation of model 1 by model 2 for which the KL divergence $\operatorname{KL}[p(\theta, z \mid \gamma, \phi), q(\theta, z \mid \boldsymbol{w}, \alpha, \beta)$ is minimal

- Iterative updating of $\gamma$ and $\phi$ for each document and recalculation of corpus-level variables $\alpha$ and $\beta$ by means of EM algorithm

- Inference for new document:

- Given $\alpha$ and $\beta$ : we determine $\gamma$ (topic distribution) and $\phi$ (word distribution) with a variational inference algorithm 


\section{Concept recognition}

- Concept recognition in text, images, ... :

- When examples annotated with concepts are available:

- Training of classifier: super vector machine, maximum entropy model, ...

- Unsupervised: extensions of probabilistic topic models 


\section{Expert recognition}

- Experts = persons named with strong correlation to certain topics

- Expert recognition requires:

- Named entity recognition

- Topic detection 


\section{Linking of content}

- Here restricted to linking of person mentions:

- In one document: anaphora resolution and noun phrase coreference resolution includes mention clustering and disambiguation

- Across documents: noun phrase coreference resolution includes mention clustering and disambiguation

- Across media: alignment of names and faces 
Table 4.2. Typical features in a single-document noun phrase coreference, resolution task of the syntactic heads, $i$ and $j$, of two candidate coreferent noun phrases in text $T$ where $i<j$ in terms of word position in $T$.

围

\begin{tabular}{|l|l|l|}
\hline FEATURE & $\begin{array}{l}\text { VALUE } \\
\text { TYPE }\end{array}$ & VALUE \\
\hline $\begin{array}{l}\text { Number } \\
\text { agreement }\end{array}$ & Boolean & $\begin{array}{l}\text { True if } i \text { and } j \text { agree in number; False other- } \\
\text { wise. }\end{array}$ \\
\hline $\begin{array}{l}\text { Gender } \\
\text { agreement }\end{array}$ & Boolean & True if $i$ and $j$ agree in gender; False otherwise. \\
\hline Alias & Boolean & $\begin{array}{l}\text { True if } i \text { is an alias of } j \text { or vice versa; False oth- } \\
\text { erwise. }\end{array}$ \\
\hline Weak alias & Boolean & $\begin{array}{l}\text { True if } i \text { is a substring of } j \text { or vice versa; False } \\
\text { otherwise. }\end{array}$ \\
\hline POS match & Boolean & $\begin{array}{l}\text { True if the POS tag of } i \text { and } j \text { match; False oth- } \\
\text { erwise. }\end{array}$ \\
\hline Pronoun $i$ & Boolean & True if $i$ is a pronoun; False otherwise. \\
\hline Pronoun $j$ & Boolean & True if $j$ is a pronoun; False otherwise. \\
\hline Appositive & Boolean & True if $j$ is the appositive of $i$; False otherwise. \\
\hline Definiteness & Boolean & $\begin{array}{l}\text { True if } j \text { is preceded by the article "the" or a } \\
\text { demonstrative pronoun; False otherwise. }\end{array}$ \\
\hline $\begin{array}{l}\text { Grammatical } \\
\text { role }\end{array}$ & Boolean & $\begin{array}{l}\text { True if the grammatical role of } i \text { and } j \text { match; } \\
\text { False otherwise. }\end{array}$ \\
\hline Proper names & Boolean & $\begin{array}{l}\text { True if } i \text { and } j \text { are both proper names; False } \\
\text { otherwise. }\end{array}$ \\
\hline $\begin{array}{l}\text { Named entity } \\
\text { class }\end{array}$ & Boolean & $\begin{array}{l}\text { True if } i \text { and } j \text { have the same semantic class } \\
\text { (e.g., person, company, location); False other- } \\
\text { wise. }\end{array}$ \\
\hline $\begin{array}{l}\text { Discourse } \\
\text { distance }\end{array}$ & Integer $>0$ & $\begin{array}{l}\text { Number of sentences or words that } i \text { and } j \text { are } \\
\text { apart. }\end{array}$ \\
\hline & & \\
\hline
\end{tabular}




\section{Pairwise coreference classifier}

- $\mathbf{m}$ is a set of mentions $\left(m_{i}\right)$ (e.g., noun phrases) in the document

- $\mathbf{x}$ is the set of pairs of noun phrases: $x_{i j}=\left\{m_{i}, m_{j}\right\}$

- $\mathbf{y}$ is the set of variables representing each pairwise coreference decision $y_{i j}$ involving mentions $m_{i}$ and $m_{j}$

- Binary random variable $y_{i j}=1$ if $m_{i}$ and $m_{j}$ are coreferent

- Let $F=\left\{x_{i j,} y_{i j}\right\}$ be a set of feature functions over $x_{i j}$ (e.g., Boolean)

- $p\left(y_{i j} \mid x_{i j}\right)$ : computed with a classification model 


\section{Pairwise coreference classifier}

- Maximum entropy model (i.e., (multinomial) logistic regression):

$$
p\left(y_{i j} \mid x_{i j}\right)=\frac{1}{Z} \exp \left(\sum_{k} w_{l} f_{l}\left(x_{i j}, y_{i j}\right)\right), 0<w_{l}<\infty
$$

$f_{(}\left(x_{i j}, y_{i j}\right)=$ one of the $k$ binary-valued feature functions

$w_{l}=$ real-valued weight parameter estimated from the training data

$Z=$ normalizing constant 


\section{Constructing the clusters}

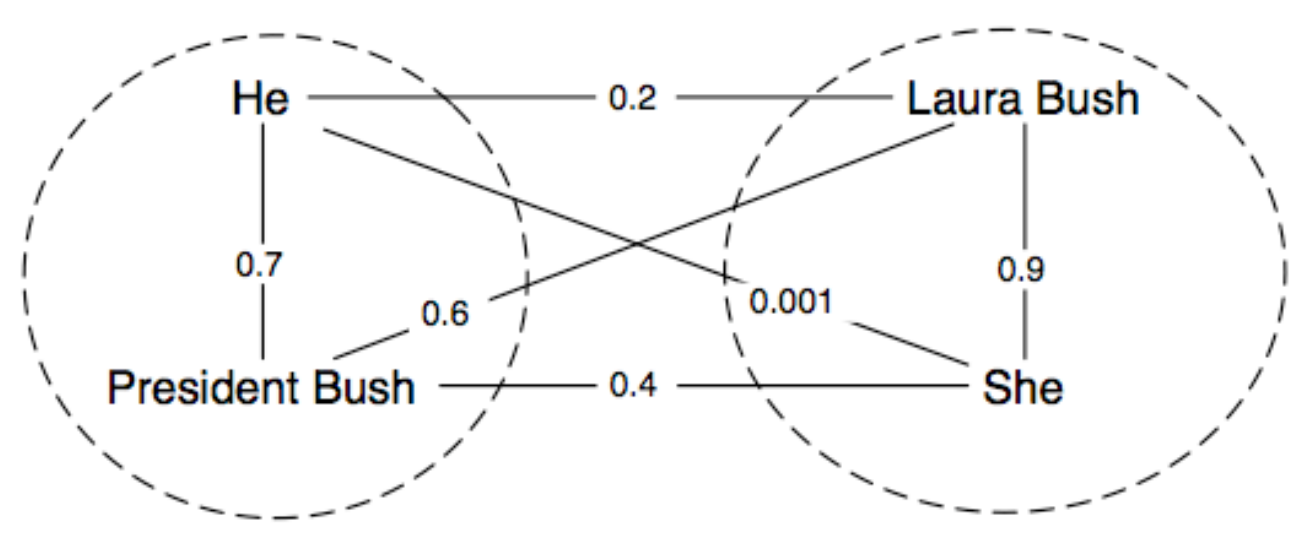

Figure 1: An example noun coreference graph in which vertices are noun phrases and edge weights are proportional to the probability that the two nouns are coreferent. Partitioning such a graph into disjoint clusters corresponds to performing coreference resolution on the noun phrases. 


\section{Constructing the clusters}

- Goal: partition graph into clusters with high intra-cluster edge weights and low inter-cluster edge weights:

- Based on strength (or probability) of detected relations

- Possibly augmented with heuristic constraints of forbidden merge of mentions

- Often greedy clustering: each noun phrase $m_{i}$ is assigned to the same cluster as the closest preceding noun phrase $m_{j}$ for which $p\left(y_{i j} \mid x_{i j}\right)>\delta$ (threshold) (e.g., $\delta$ $=0.5$ ) 


\section{Constructing the clusters}

- How to incorporate information over sets of noun phrases? Some alternatives:

- Best clustering: intractable problem

- At least incorporate transitivity constraints 


\section{Enforcing transitivity with ILP}

- After computing the pairwise classification decisions:

- Use integer linear programming to enforce transitivity constraints:

$$
\max \sum_{x_{i j}} \log p\left(y_{i j} \mid x_{i j}\right) \cdot y_{i j}-\log \left(1-p\left(y_{i j} \mid x_{i j}\right)\right) \cdot\left(1-y_{i j}\right)
$$

- $p\left(y_{i j} \mid x_{i j}\right)$ : computed with a classification model (see above) 


\section{Enforcing transitivity with ILP}

- Add binary constraints on each of the variables: $y_{i j}$ $\in\{0,1\}$

- Add constraints over each triplet of mentions to enforce transitivity: $\left(1-y_{i j}\right)+\left(1-y_{j k}\right) \geq\left(1-y_{i k}\right)$

- ensures that whenever $y_{i j}=y_{j k}=1$ also $y_{i k}=1$

- Use ILP tool to solve the ILP optimization problem

- Solution for short text because of computational complexity of ILP 


\section{Linking of entities across documents}

- 2 problems:

- Homonymy = names haves the same writing, but refer to different entities:

-E.g., persons disambiguation on the Web, cf. Web People Search Task (WePs)

- Synonymy = names are written differently, but refer to the same entity (cf. within document noun phrase coreference resolution, but "one sense per discourse" heuristic not applicable):

-E.g., people hide their identity in different names or names might have different writing formîs 


\section{Linking of entities across documents}

- In both cases the context is important:

- Context determines whether two mentions refer to the same entity or to different ones

- Context:

- Surrounding words

- Other entities mentioned in close vicinity

- Other linked information 


\section{Homonymy}

- Most simple problem: given a name only disambiguation

- Approaches:

- Feature vector represents potential coreference relationship

- Usually supervised:

- Training of classifier

- Clustering of the candidate coreference relationships possibly via graph partitioning 
Table 4.3. Typical features in a cross-document noun phrase coreference resolution task of the syntactic heads, $i$ and $j$, of two candidate coreferent noun phrases where $i$ and $j$ occur in different documents.

\begin{tabular}{|l|l|l|}
\hline FEATURE & TYPE & VALUE \\
\hline Context word & $\begin{array}{l}\text { Boolean or } \\
\text { real value } \\
\text { between } 0 \text { and } \\
1\end{array}$ & $\begin{array}{l}\text { True if the context word } x \text { occurs in the context } \\
\text { of } i \text { and } j \text {; False otherwise; If a real value is } \\
\text { used, it indicates the weight of the context } \\
\text { word; Proper names, time and location } \\
\text { expressions in the context might receive a high } \\
\text { weight. }\end{array}$ \\
\hline $\begin{array}{l}\text { Named entity } \\
\text { class }\end{array}$ & Boolean & $\begin{array}{l}\text { True if } i \text { and } j \text { have the same semantic class } \\
\text { (e.g., person, company, location); False } \\
\text { otherwise. }\end{array}$ \\
\hline $\begin{array}{l}\text { Web-related } \\
\text { information }\end{array}$ & Boolean & $\begin{array}{l}\text { True if URL or email address } x \text { occurs in the } \\
\text { context of } i \text { and } j \text {; False otherwise. }\end{array}$ \\
\hline Semantic role & Boolean & $\begin{array}{l}\text { True if the semantic role of } i \text { matches the } \\
\text { semantic role of } j ; \text { False otherwise. }\end{array}$ \\
\hline
\end{tabular}




\section{Synonymy}

- Given a name, find synonyms AND also disambiguation

- String edit distances might not be sufficient

- Graph based approaches: link based similarity measures between nodes (exploiting similarity of neighbors), e.g.,

- Co-citation

- SimRank, Connected-Triple, PageSim

- Variety of random-walk methods

[Getoor \& Diehl ACM SIGKDD Explorations 2005] [Liben-Nowell \& Kleinberg JASIST 2007] 


\section{Alignment across media}

- Novel area of research, currently mainly focusing on aligning names and faces

- Helps in automatically annotating images, video, ... 


\section{Alignment of names and faces}

- Given image-text pairs: align faces $(f)$ and names $(n)$

- Constraints:

- Within image/text resolution of a face/name => same name and face occur once in each image/text

- One name can only be aligned with one face,

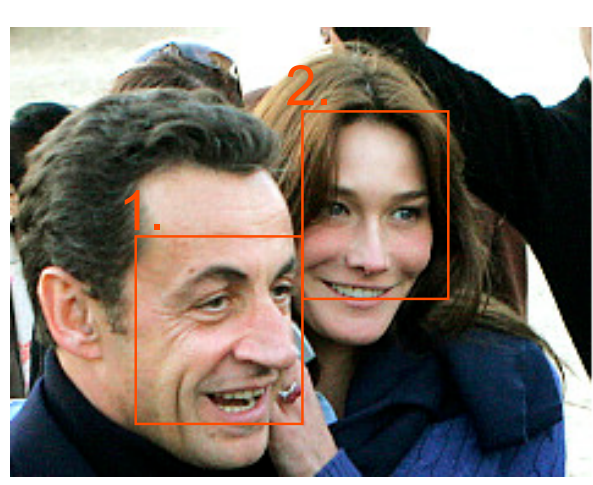
but faces can be aligned with "null" name and names with "null" face

French President 1. Nicolas

Sarkozy and girlfriend 2. Carla

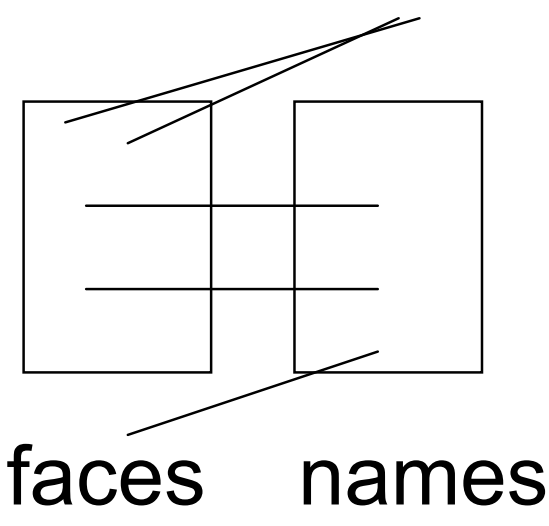

Bruni on a trip in Egypt...

[Pham et al. ECCV Workshop o chlor 


\section{Preprocessing}

- Images:

- face detection

- clustering of similar faces (within and) across images (based on face descriptors)

- computation of the namedness of the faces

- Texts:

- named entity (person) recognition: maximum entropy classifier augmented with gazetteers

- clustering of similar names within and across texts: noun phrase coreference resolution

- computation of the picturedness of the names 
Cardinal from Cologne Joachim Meisner cries during a meeting with Pope Benedict XVI at the centre for dialog and prayer in Oswiecim, Poland May 28, 2006.

$<$ ?xml version="1.0" encoding="UTF-8"?><output $><$ s i="0">Cardinal from Cologne <ENAMEX ID="0" TYPE="PERSON">Joachim Meisner</ENAMEX> cries during a meeting with Pope <ENAMEX ID="1" TYPE="PERSON">Benedict</ENAMEX> XVI at the centre for dialog and prayer in <ENAMEX ID="2" TYPE="LOCATION">Oswiec im</ENAMEX>, <ENAMEX ID=" 3" TYPE="LOCATION">Poland</ENAMEX> May 28, $2006 .</ s>$

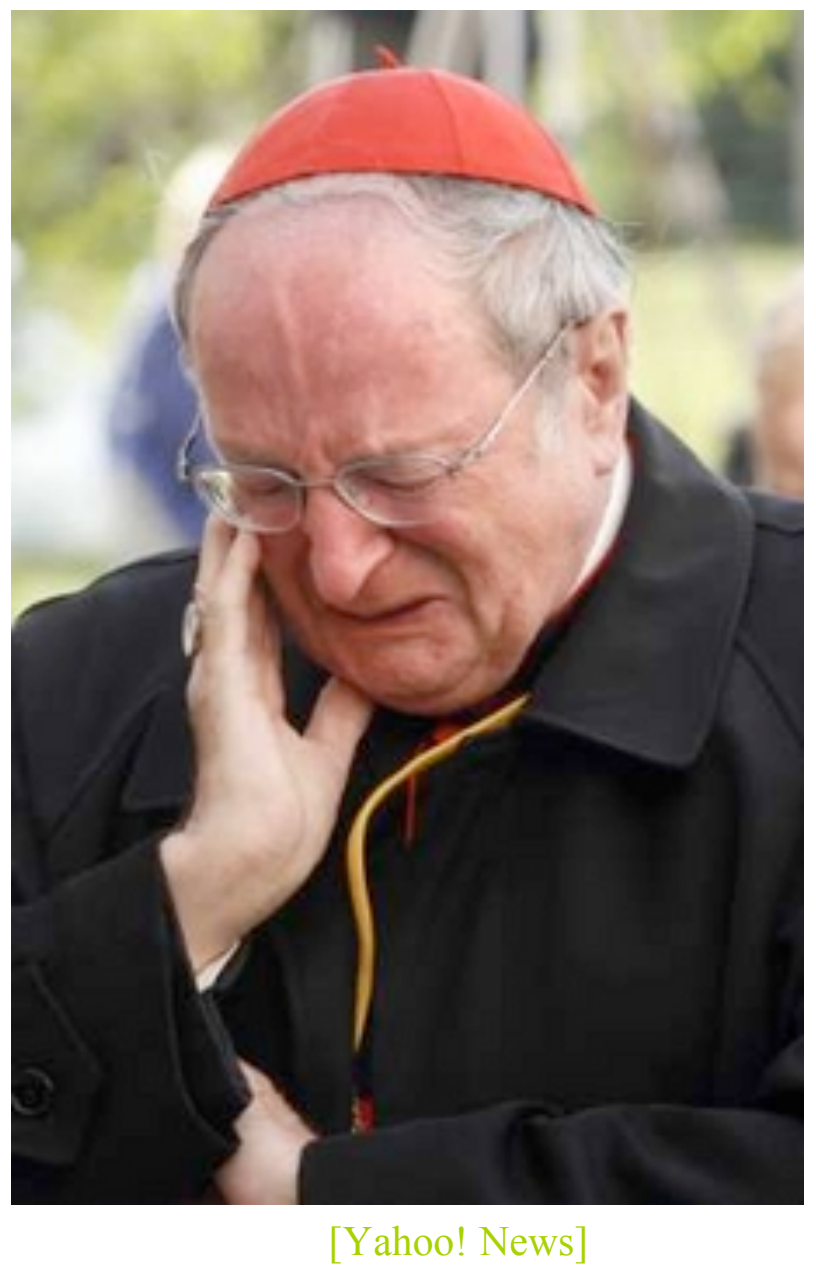

Picturedness of name:

Joachim Meisner: 0.75

Benedict: 0.33 


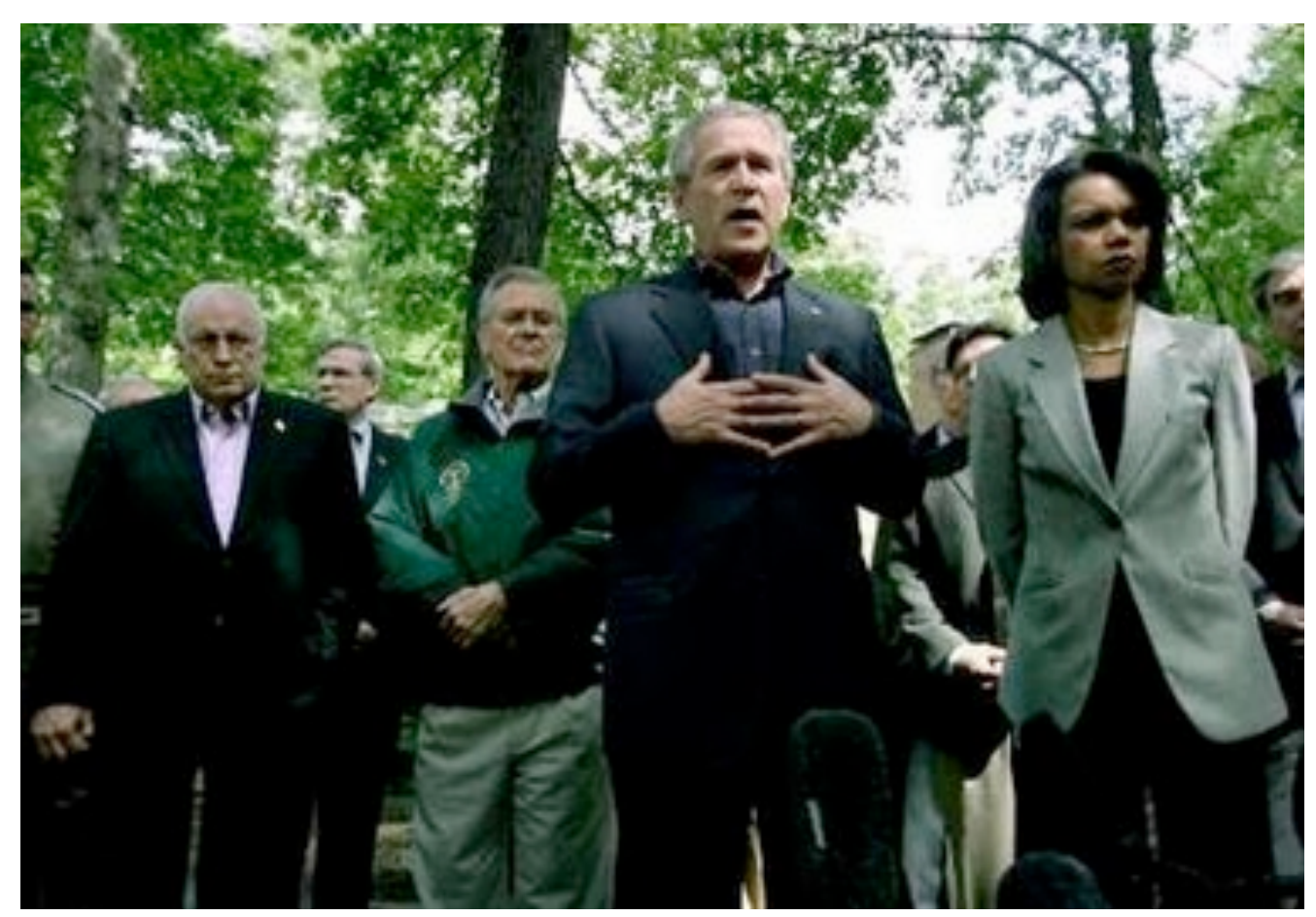

[Yahoo! News]

U.S. President George W. Bush (2nd R) speaks to the press following a meeting with the Interagency Team on Iraq at Camp David in Maryland, June 12, 2006. Pictured with Bush are (L-R) Vice President Dick Cheney, Defense Secretary Donald Rumsfeld and Secretary of State Condoleezza Rice.

Namedness of face: is proportional with size of detected face and confidence of detection 
Likelihood of image-text pair $x_{i}$ and the alignment $a_{j}$ :

$$
\begin{aligned}
& L_{x_{i}, a_{j}}^{(n \rightarrow f)}= \prod_{\alpha} P\left(f_{\sigma(\alpha)} \mid n_{\alpha}\right) \\
& L_{x_{i}, a_{j}}^{(f \rightarrow n)}= \prod_{\beta} P\left(n_{\sigma(\beta)} \mid f_{\beta}\right) \\
& L_{x_{i}, a_{j}}^{\left(n^{*} \rightarrow f\right)}= \prod_{\alpha, \sigma(\alpha) \neq N U L L}\left(P\left(\text { pictured }_{\alpha} \mid t_{x_{i}}\right)\right. \\
&\left.P\left(f_{\sigma(\alpha)} \mid n_{\alpha}\right)\right)
\end{aligned}
$$




\section{Initialization}

- Different methods for initial parameter estimation:

- Based on one document/story: e.g., use of picturedness or namedness value

- Use of face/name clusters combined with cooccurrence information of names and faces 


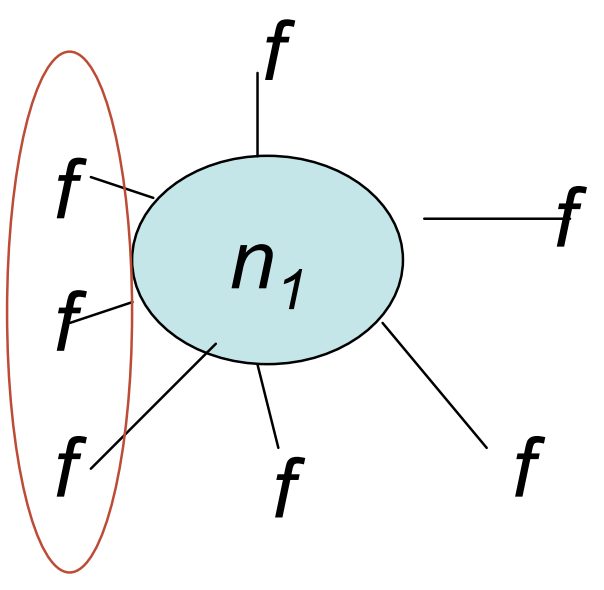

e.g., estimating $P(f \mid n)$
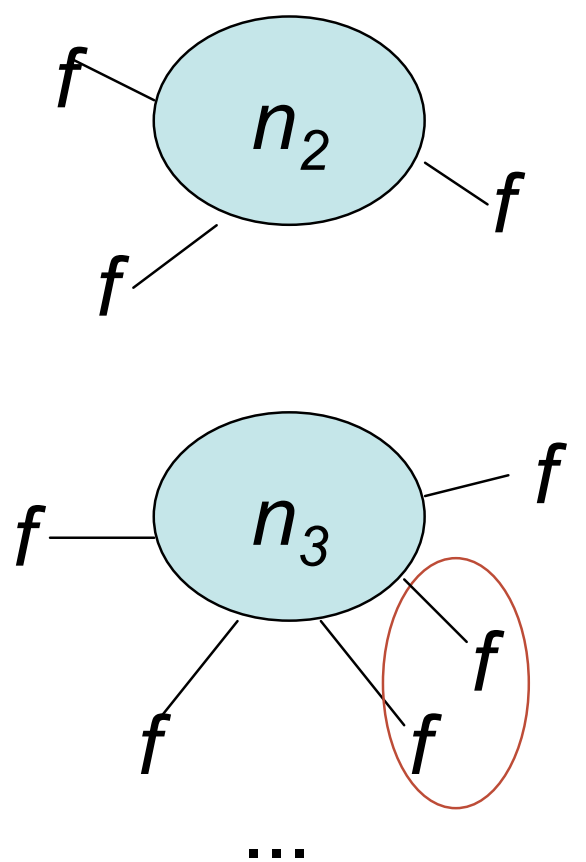

트임ㅁㅇㅛ 


\section{Optimization}

- Use of the EM algorithm to maximize the loglikelihood of all image-text pairs $S$ :

$$
\sum_{x i \in S a_{j} \in C_{i}} \delta_{i, j} \log \left(L_{\left(x i, a_{j}\right)}\right)
$$

where $C_{i}=$ set of all possible alignments for imagetext pair $x_{i,} \delta_{i, j}=$ strength of the alignment $a_{j}$ for imagetext pair $x_{i}$ 
- "Faces in the wild" dataset: 11820 stories or image-text pairs with 5637 unique person faces and 8878 unique person names.

- Best alignment results: $72.33 \% \mathrm{~F}_{1}$ measure 


\section{References}

- Abbasi, A., Chen, H. \& Salem, A. (2008). Sentiment analysis in multiple languages. ACM Transactions on Information Systems, 26 (3), article 12.

- Berg, T.L., Berg, A.L., Edwards, J. \& Forsyth, D.A. (2004). Who's in the picture? Information Processing Systems (NIPS).

- Berger, A., Della Pietra, S.A. \& Della Pietra, V.J. (1996). A maximum entropy approach to natural language processing. Computational Linguistics, 22 (1), 3971.

- Blei, D.M., Ng, A.Y. \& Jordan, M.I. (2003). Latent Dirichlet allocation. Journal of Machine Learning Research, 3, 993-1022.

- Boiy, E. \& Moens M.-F. (2009). A machine learning approach to sentiment analysis in multilingual Web texts. Information Retrieval, 30 p. SpringerLink

- Culotta, A., Wick, M., Hall, R. \& McCallum, A. (2007). First-order probabilistic models for coreference resolution. In Proceedings of HLT/NAACL.

- Deerwester, S., Dumais, S.T., Furnas, G.W., Landauer, T.K. \& Harshman, R. (1990). Indexing by latent semantic analysis. Journal of the American Society for Information Science, 41 (6), 391-407.

- Deschacht, K. \& Moens, M.-F. (2007). Text analysis for automatic image annotation. In Proceedings of the 45th Annual Meeting of the Association for Computational Linguistics, Prague, Czech Republic, June 23th-30th, 2007. 
- Hofmann, T. (1999). Probabilistic latent semantic indexing. In Proceedings of Twenty-second Annual International ACM-SIGIR Conference on Research and Development in Information Retrieval.

- Finkel, J.R. \& Manning, C.M. (2008). Enforcing transitivity in coreference resolution. In Proceedings of $A C L / H L T$.

- Getoor, L. \& Diehl , C.P. (2005). Link mining: a survey. ACM SIGKDD Explorations Newsletter, 7 (2), 3-12.

- Kalashnikov, D.V., Chen, Z., Mehrotra, S. \& Nuray-Turan, R. (2008). Web people search via connection analysis. IEEE Transactions on Knowledge and Data Engineering, 20 (11).

- Krishnan, V. \& Manning, C.D. (2006). An effective two-stage model for exploiting non-local dependencies in named entity recognition. Proceedings of COLINGACL 2006 (pp. 1121-1128). East Stroudsburg, PA: ACL.

- Lafferty, J., McCallum, A. \& Pereira, F.C.N. (2001). Conditional random fields: Probabilistic models for segmenting and labelling sequence data. In Proceedings of the $18^{\text {th }}$ International Conference on Machine Learning (pp. 282289). San Francisco, CA: Morgan Kaufmann.

- Liben-Nowell, D. \& Kleinberg, J. (2007). The link prediction problem for social networks. Journal of the American Society for Information Science and Technology, 58 (7), 1019-1031. 
- Moens, M.-F. (2006). Information Extraction in a Retrieval Context (The Information Retrieval Series 21). New York: Springer.

- Pham, P.T., Moens, M.-F. \& Tuytelaars, T. (2008). Linking names and faces: Seeing the problem in different ways. In Proceedings of ECCV Workshop on Faces in Real-Life Images: Detection, Alignment and Recognition.

- Pham, P.T., Moens, M.-F. \& Tuytelaars, T. (2009). Cross-media alignment of names and faces. 14 p. (submitted).

- Steyvers, M. \& Griffiths, T. (2007). Probabilistic topic models. In T. Landauer, D. McNamara, S. Dennis \& W. Kintsch (Eds.), The Handbook of Latent Semantic Analysis. Lawrence Erlbaum Associates. 